\title{
ANALYSIS OF ANTIGENIC RELATIONSHIPS AMONG INFLUENZA VIRUS STRAINS USING A TAXONOMIC CLUSTER PROCEDURE. COMPARISON OF THREE KINDS OF ANTIBODY PREPARATIONS
}

\author{
T.F. WEIJERS', A.D.M.E. OSTERHAUS', W.E.P. BEYER'2. J.A.A.M. VAN ASTEN', F.M. DE \\ RONDE-VERLOOP', K. BIJLSMA' and J.C. DE JONG'
}

'Rijksinstituut voor Volksgezondheid en Milieuhygiene, Bilthoven; and ${ }^{2}$ Erasmus University, Rotterdam. The Netherlands

(Accepted 31 October 1984)

Hemagglutination inhibiting (HI) monoclonal antibody preparations (MA) were raised against six influenza $A(H 3 N 2)$ strains from the period 1977-1982. Twenty-three hybridomas were selected and titrated in $\mathrm{HI}$ assays against these strains and against 18 influenza $A(H 3 N 2)$ viruses isolated in The Netherlands during the seasons 1981-1982 and 1982-1983. Similar HI tests were performed with conventional post-infection ferret antisera and with ferret antisera adsorbed with heterologous strains of influenza A (H3N2) virus. The resulting serological data were subjected to a computerized taxonomic cluster procedure based on the Euclidean distance between viruses. With respect to the degree of separation between clusters the unadsorbed ferret antisera were inferior to the adsorbed antisera whereas the MA were superior to both. Our results demonst rate that computer programs based on numerical taxonomy can be helpful in processing large numbers of serological data and that $\mathrm{MA}$ are indispensable in epidemiological and diagnostic influenza studies.

influenza virus numerical taxonomy monoclonal antibodies

\section{INTRODUCTION}

The large antigenic variability of influenza A virus, especially of its hemagglutinin (HA), is of great epidemiological importance. It necessitates a continuous virus surveillance and a yearly evaluation of the vaccine strains in use (Anon., 1979). Almost yearly small antigenic changes are noticed, which constitute the so-called 'antigenic drift' (Webster et al., 1982; Skehel et al., 1982). This antigenic drift is monitored mainly by comparing newer and older virus isolates in HI tests. Introduced 50 yr ago by Smith and coworkers (1933), post-infection ferret antisera still constitute a major tool in such tests. In fact, the only accepted way to determine whether an isolate should be considered a new strain is based on the demonstration of differences in reactivity between isolate and reference strains in $\mathrm{HI}$ assays, using ferret antisera as antibody preparations (Dowdle et al., 1979).

So far, MA have predominantly been used to investigate the 'molecular epidemiolo- 
gy' of influenza A, e.g. to select variants of HA (Nakajima and Kendal, 1981) and to characterize the antigenic topology of the HA molecule (Gerhard et al., 1981). However, in diagnostic and epidemiological routine their use has been quite limited. After adsorption with hetcrologous strains, ferret antisera show increased specificity (Takatsy and Hamar, 1955). For this reason they are routinely used in one of our laboratories (RIVM, Bilthoven) for typing and comparison of influenza A virus strains (De Jong et al., 1982).

The objective of this study was to assess the relative usefulness of the three kinds of antibody preparations mentioned for the characterization and discrimination of recent influenza $A(\mathrm{H} 3 \mathrm{~N} 2)$ virus isolates from The Netherlands. To facilitate this comparison, and to analyse larger series of similar serological data in the future, a computer program was developed on the basis of the Theoretical Pattern Fitting (TPF) program we previously devised for the characterization of poliovirus strains (Osterhaus et al., 1983).

\section{MATERIALS AND METHODS}

\section{Viruses}

Six influenza A (H3N2) strains from the period 1977-1982 were selected for the production of $\mathrm{MA}$ and of adsorbed and unadsorbed ferret antisera, namely: A/Texas/1/77, A/Bangkok/1/79, A/Bangkok/2/79, A/England/496/80, A/Shanghai/31/80 and $\mathrm{A} /$ Philippines $/ 2 / 82$. These strains will be referred to hereafter as 'reference strains'.

The reference strains were obtained from the WHO Influenza Centre in London (Dr. J.J. Skehel) and were propagated in embryonated fowl eggs and in tertiary cynomolgus monkey kidney cells (kindly provided by Dr. A.L. van Wezel, Bilthoven, The Netherlands). Eighteen recent influenza A (H3N2) virus isolates from The Netherlands (designated: 1-18) obtained during the influenza seasons 1981-1982 (isolates 1-8) and 1982-1983 (isolates 9-18), were propagated in tertiary monkey kidney cells.

\section{Production of ferret antisera}

Ferret antisera were raised against the six reference strains mentioned above and against isolate 14 (A/Netherlands/241/82) by intranasal infection with egg-grown virus and bleeding after 18 days. Adsorption of ferret antisera was carried out as previously described (De Jong et al., 1982) with the following heterologous strains:

Ferret antiserum against: Adsorbed with:

A $/$ Texas $/ 1 / 77$

A/Bangkok/1/79

A/Bangkok/2/79

A/England/496/80

A/Shanghai/31/80
A/Bangkok/1/79 $(3 \times)$

A/Bangkok/2/79 $(3 \times)$

A/Bangkok/1/79 $(4 \times)$

A/Texas/1/77 $(3 \times)$

A/Texas/1/77 $(1 \times)$ 


\section{HI tests}

HI tests were performed as described elsewhere (De Jong et al., 1982). In short, ferret antisera and ascitic fluids were treated with cholera filtrate and incubated in serial twofold dilutions for ferret antisera and in scrial tenfold dilutions for MA with four HA units of virus in microtiter plates for $1 \mathrm{~h}$ at $37^{\circ} \mathrm{C}$. HI titers were read after addition of $0.5 \%$ fowl erythrocytes and incubation for $1 \mathrm{~h}$ at $4^{\circ} \mathrm{C}$ and were expressed as the reversed maximum dilution of the antiserum that completely inhibited HA. All tests were repeated and the resulting geometric means are presented in this paper.

\section{Production of hybridomas}

Balb/c mice were infected intranasally with 1,000 HA units of egg-grown virus. After 8-12 wk they were boosted intravenously with 500 HA units of sucrose gradicntpurified virus on 3 consecutive days. Two days after the last booster their splenocytes were removed and fused with 8-azaguanine-resistant P3/X63 Ag8-653 mouse myeloma cells, using the method developed by Köhler and Milstein (1975) with minor modifications (Osterhaus et al., 1981). After fusion, cells were resuspended in HAT medium and seeded in eight 24-well cell culture plates (Cat. no. 704160, Greiner, Nürtingen, F.R.G.), using rat thymocytes as feeder cells. Culture fluids from the fused cells were screened in HI assays. HI-positive cultures were selected and cloned twice by limiting dilution. Of cach cloned hybridoma $1-2 \times 10^{6}$ cclls were injected intraperitoneally into pristane-treated mice. Between 1 and 3 wk later, between 2 and $10 \mathrm{ml}$ of ascitic fluid were obtained per animal and these fluids were used in HI tests. Six fusions, following the immunization of $\mathrm{Balb} / \mathrm{c}$ mice with the six reference strains mentioned above, resulted in the production of over 300 hybridomas. Twenty-three of these were finally selected, aiming at MA which showed weak or no reactions with heterologous reference strains, or showed HI patterns different from the other selected MA.

\section{Calculations}

To establish the Euclidean distance coefficient $d^{2}$ between two virus strains, for each panel the squared differences between the ${ }^{10}$ logarithms of the titers of each antibody preparation tested against the two strains were summated, and the sum was divided by the total number of antibody preparations involved. As a consequence of this definition, a $d^{2}$ value of zero would mean identity of the two strains with respect to the antibody preparations used. In this way, each of the 24 strains was compared with all other 23 strains, resulting in a matrix of 552 coefficients for each panel of antibodies (MA, adsorbed and unadsorbed ferret antisera).

For the calculations a UNIX operated PDP 1170 computer was employed. This computer was programmed with Fortran 77 for the cluster analysis. The program was constructed to work interactively with data files. This implies that the titers were read for one strain at a time. Selected patterns as well as relevant data were stored on predefined files. 
For each panel of antibodies, the distance coefficients were subjected to a taxonomic cluster procedure: the weighted pair-group method using arithmetic averages, which is described in detail by Sokal and Sneath (1963). These calculations were done on a Hewlett-Packard $41 \mathrm{CV}$ computer. Dendrograms were drawn to illustrate the obtained cluster structure for each panel of antibodies.

\section{RESULTS}

\section{Construction of dendrograms}

Twenty-four influenza A (H3N2) virus strains, namely six reference strains from WHO and 18 isolates from The Netherlands, were tested in $\mathrm{HI}$ assays against three panels of antibody preparations, i.e. 7 post-infection ferret antisera, 5 cross-adsorbed ferret antisera and $23 \mathrm{MA}$. As expected the apparent degree of relatedness among the strains varied widely from one antibody preparation to the other (Table 1). To express the relatedness between any pair of strains by means of only one number, we developed a computerized method as described in Materials and Methods. This computer program, an extension of the TPF program which was first used for the identification of poliovirus strains (Osterhaus et al., 1983), can store large numbers of strains with up to $160 \mathrm{HI}$ titers for each strain and offers the following options:

(1) for each virus isolate: calculation of the Euclidean distance coefficient $d^{2}$ relative to every other virus strain; selection of the most resembling strain;

(2) calculation of an average HI titer pattern for a group of related virus strains.

With the aid of this method we constructed Euclidean distance coefficient matrices. As an example, the values of $d^{2}$ are shown for the MA (Table 2). Subsequently, these matrices were worked up into dendrograms, which quantitatively picture the network of relationships among the strains for each of the three antibody panels (Fig. 1).

\section{Antigenic relations among reference strains}

The HI reactivity of the six reference strains showed minor and major differences with all antibody preparations used (Table 1). The MA demonstrated large differences, showing values of $d^{2}>1.82$ except for the pair $\mathrm{A} / \mathrm{Bangkok} / 1 / 79-\mathrm{A} / \mathrm{Shang}$ hai/31/80 with $d^{2}=0.31$ (Table 2 and Fig. 1). The cross-adsorbed ferret antisera discriminated less well than the MA ( $d^{2}$ from 0.15 to 1.19 ) and their usefulness was further lessened by their low homologous titers (Table 1 and Fig. 1). Finally, the unadsorbed ferret antisera were the least discriminative, displaying values of $d^{2}$ from 0.14 to 0.60 (Tablc 1 and Fig. 1).

\section{Antigenic relations among virus isolates from The Netherlands}

In $\mathrm{HI}$ tests the 18 virus strains from The Netherlands reacted quite uniformly (Table 1). Only isolate 4 appeared different. This impression was substantiated in the dendrograms (Fig. 1). Seventeen of the isolates were found to be closely related $\left(d^{2}<0.12\right)$. Isolate 4 set itself apart with values of $d^{2}$ of 0.71 and 0.98 against the 


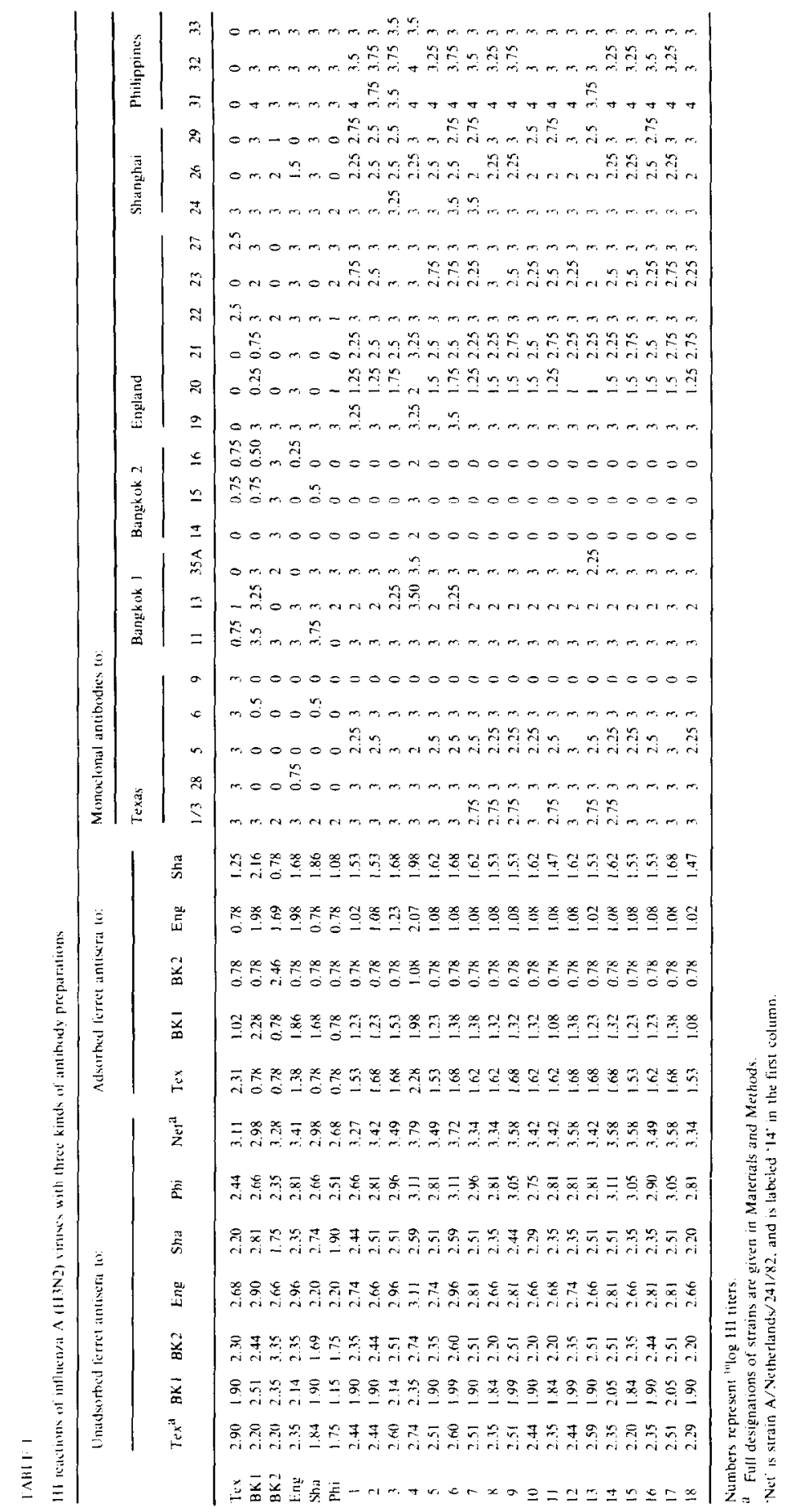




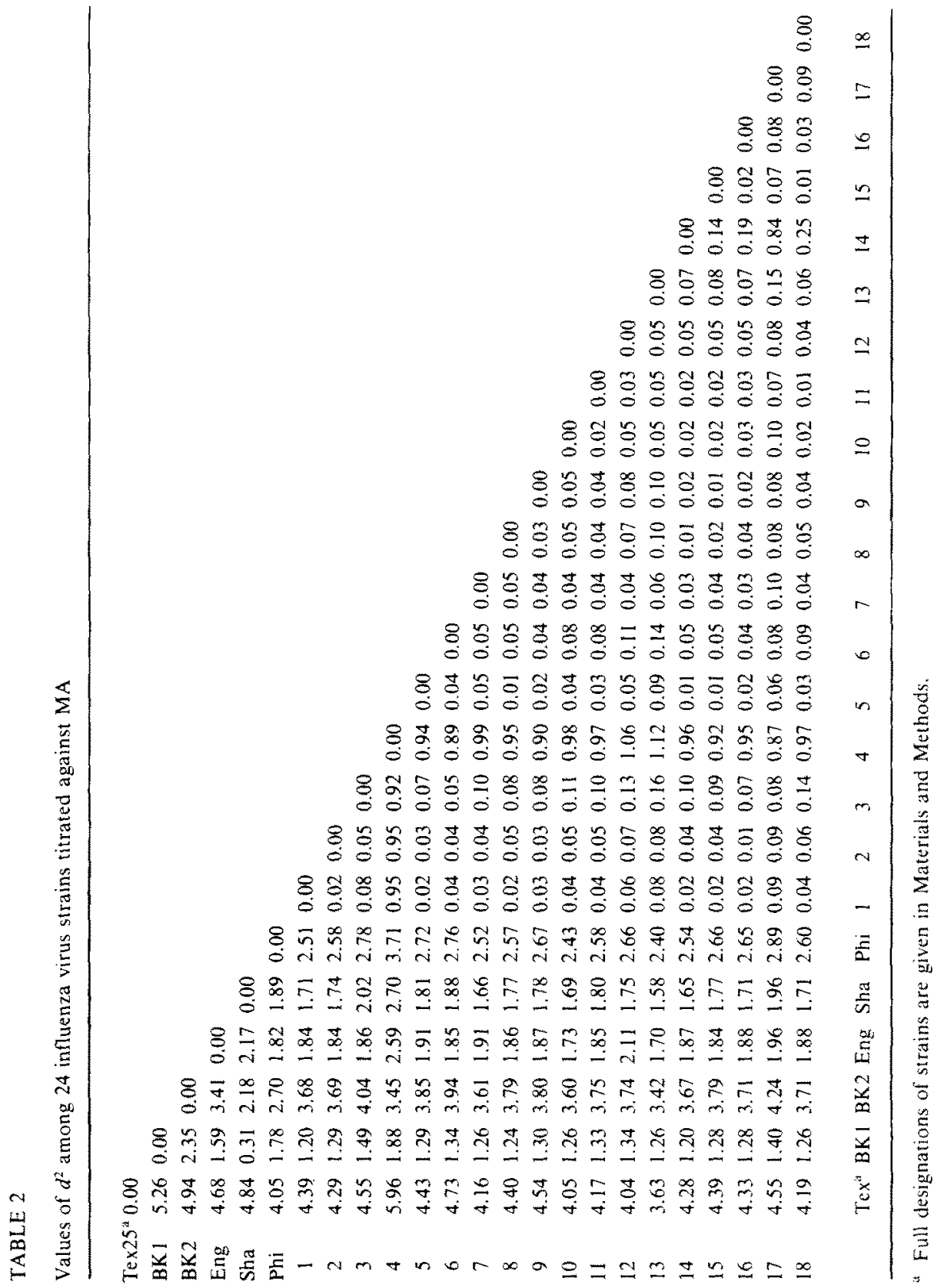



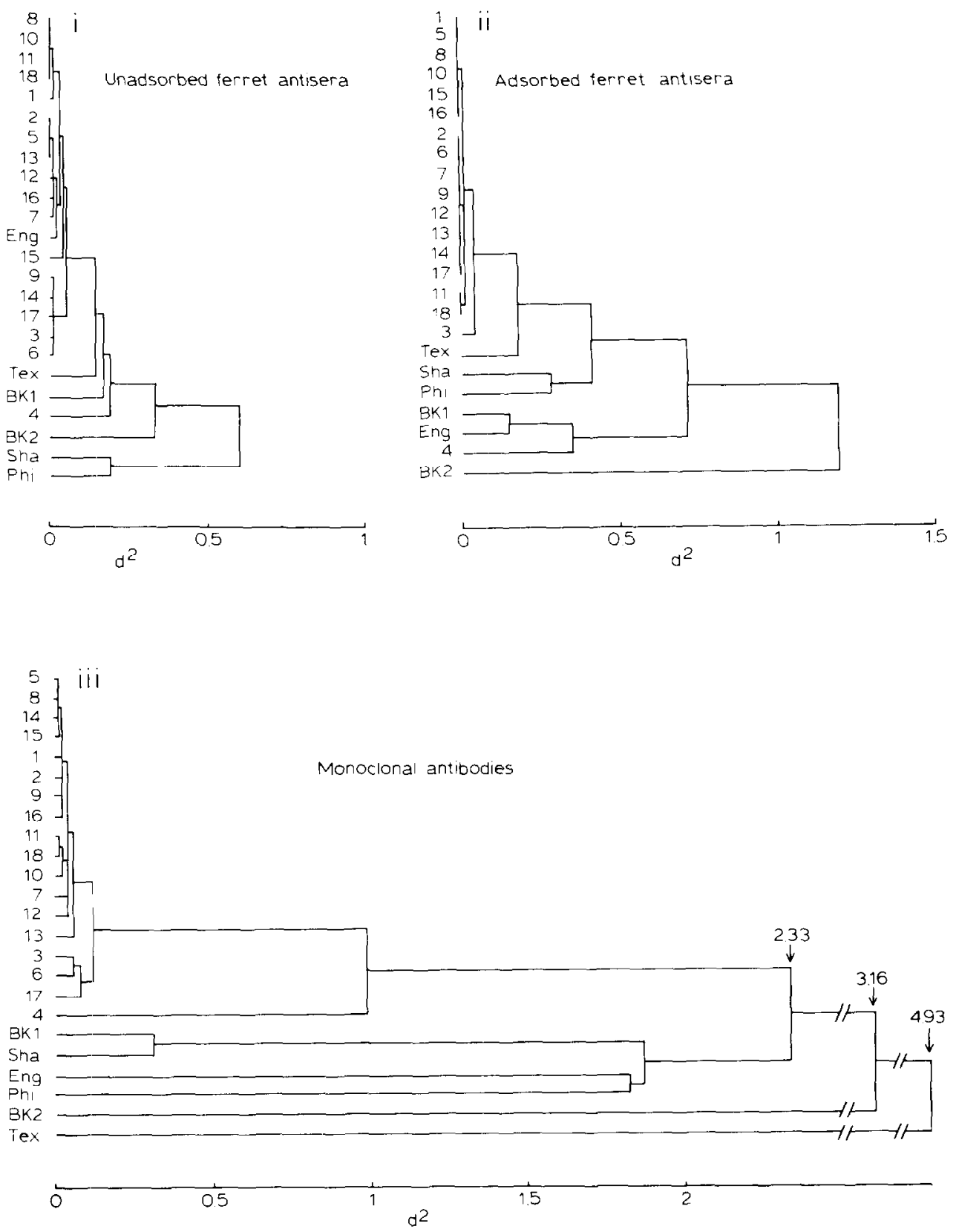

Fig. 1. Dendrograms based on Euclidean distance coefficients $\left(d^{2}\right)$ of six reference strains and 18 isolates from The Netherlands, titrated against panels of i) unadsorbed ferret antisera, ii) cross-adsorbed ferret antisera, and iii) MA. 


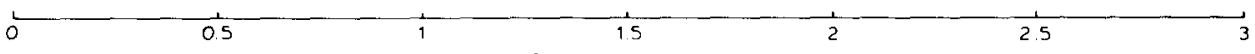

Fig. 2. Differentiating ability of panels of unadsorbed and cross-adsorbed ferret antisera and MA, depicted as the Euclidean distance coefficient $\left(d^{2}\right)$. Given are the mean $(0)$ and the root mean square deviation (— ) of the $d^{2}$ values i) among the 17 closely related isolates from The Netherlands (left), and ii) among all 24 strains (right).

cross-adsorbed ferret antisera and the MA, respectively. As with the reference strains, the unadsorbed ferret antisera differentiated least. They even included reference strain A/England/496/80 in the group of 17 isolates.

Comparison of the discriminative abilities of the three kinds of antibody preparations

The differentiating power of the three panels of antibodies is quantitatively compared in Fig. 2. In this figure the sum of the mean and the root mean square deviation as a measure of discriminative ability is given: i) for the closely related 17 isolates from The Netherlands, and ii) for all strains. None of the three panels could detect appreciable differences among the 17 isolates (sum of mean and deviation is $0.05,0.02$ and 0.08 for unadsorbed and adsorbed ferret antisera, and MA, respectively). The spread for all strains, however, is very different $(0.27,0.56$ and 2.78 , respectively).

\section{DISCUSSION}

This study shows that for the comparison of influenza virus isolates by HI tests each of the three kinds of antibody preparations used has its own value. The unadsorbed ferret antisera demonstrated the overall relatedness. The adsorbed antisera highlighted the differences in the major immunogenic determinants. The monoclonal antibody preparations revealed the differences in individual antigenic determinants.

All three panels differentiated the viruses isolated in The Netherlands from each of the reference strains. The only exception was A/England/496/80 virus which could not be distinguished from the isolates by unadsorbed ferret antisera. Also, all three panels demonstrated 17 of 18 isolates from The Netherlands to be almost identical. Isolate 4 was shown to deviate from the other 17 isolates. The monoclonal panel did so most clearly. Strains showing the same type of reactivity as isolate 4 were detected in 
The Netherlands in the years before 1982 at a much higher frequency (De Jong, manuscript in preparation). Therefore, isolate 4 may be regarded as a 'dying' antigenic variant. Another remarkable feature of the MA dendrogram is the exceptional behaviour of the $\mathrm{A} / \mathrm{Bangkok} / 1 / 79$ and $\mathrm{A} / \mathrm{Shanghai} / 31 / 80$ reference strains. When these two strains were included in an $\mathrm{HI}$ cross titration against 23 ferret antisera they showed an almost identical reaction pattern except for a marked difference in avidity (Beyer et al., 1985). The intermediate position of these two strains in the present MA dendrogram presumably is, therefore, an expression of their different avidities.

The three panels were shown to differ greatly in discriminative power. When all strains were considered the spread was $0.27,0.56$ and 2.78 for unadsorbed and cross-adsorbed ferret antisera, and MA, respectively (Fig. 2). These numbers were based on logarithmic titers. The corresponding antilog values are 1.9, 3.6 and 602, respectively. The advantage of this larger spread displayed by the adsorbed ferret antisera and the MA is not impaired by concurrently larger spread values among the closely related 17 isolates from The Netherlands, these values being $0.05,0.02$ and 0.08 , respectively (antilog $1.12,1.05$ and 1.20 , respectively).

The question when two strains differ to an epidemiologically significant extent is difficult to answer. Often a fourfold difference in HI tests with homologous ferret antisera is used as a lower limit (Dowdle et al., 1979). This limit is not overstepped by any pair of strains among the cluster of 17 isolates from The Netherlands, when tested against the ferret antisera. The MA, however, detected differences up to a factor of six among the 17 strains. Whether or not these differences are biologically important, it is clearly impractical to consider any of such closely related viruses as a new reference strain in epidemiological studies. Perhaps, when more experience will have been acquired, quantitative criteria might be developed to define which differences in $d^{2}$ are epidemiologically significant.

It appears from this study that MA can play an important role in the epidemiological surveillance of influcnza, as was demonstrated carlicr (Webster ct al., 1979; Pcreira and Chakraverty, 1982; Yamazi et al., 1984). In addition to their relative lack of discriminative power, other drawbacks of ferret antisera are the considerable variation in antibody response among individual ferrets and the fact that high concentrations of antineuraminidase antibodies in ferret antisera may interfere in the HI test (Kilbourne et al., 1968). However, MA also have some disadvantages. Firstly, in certain serological assays they react poorly. As our selection method (HI test) for the MA was the same as the assay used for the analysis of our viruses, this disadvantage was avoided in our study. Secondly, in reactions with MA, some of the epitopes involved may be epidemiologically irrelevant. A third drawback of the routine use of MA in epidemiology is the initial workload involved, as, ideally, a fusion should be performed for every new isolate which is suspected to deviate. Ferret antisera against a new strain are easier to produce. Yet, in our opinion, these drawbacks of MA are outweighed by the convenience of having the disposal of unlimited amounts of reproducible and high-titered pure $\mathrm{HI}$ antibody preparations, which can be easily 
exchanged between laboratories. Finally, one should bear in mind that the antibody repertoires of mouse and ferret may differ, so that certain antigenic determinants recognized by either of the species are not recognized by the other.

Especially since several hybridoma banks have emerged during the last few years (Bussard, 1983), it would be feasible to provide every regional influenza laboratory functioning in the global surveillance network of the WHO with an identical panel of MA against a wide variety of strains. It was with the possibility of these future large panels of $\mathrm{MA}$ in mind that we developed a multipurpose computer program for the mathematical processing of the data which is suitable for an average personal computer and allows a rapid identification and classification of new strains.

\section{ACKNOWLEDGEMENTS}

The authors thank Mr. G. Drost and Mr. K. Siebelink for their excellent technical assistance. During part of this study T.F.W. was employed by Erasmus University, Rotterdam. Dr. N. Masurel of Erasmus University is thanked for allowing T.F.W. to take part in this study.

\section{REFERENCES}

Anon., 1979. Wrld Hlth Org. Chronicle. 33, 7.

Beyer, W.E.P. and Masurel, N., 1985, J. Hyg. (Camb.) (in press).

Bussard, A.E., 1983, Abstracts. Symposium on Monoclonal Antibodies, Standardization in their Production and Use (Institut Pasteur. Paris) p. 3.

De Jong, J.C., De Ronde-Verloop, F.M. and Dorpema, J.W., 1982, J. Biol. Stand. 10, 175.

Dowdle, W.A., Kendal A.P. and Noble, G.R., 1979, in: Diagnostic Procedures for Viral, Rickettsial and Chlamydial Infections, eds., Lennette, E.H. and Schmidt, N.J. (American Public Health Association, Washington) p. 585.

Gerhard, W., Yewdell, I., Frankel, M.F and Webster, R, 1981, Nature 290, 713

Kilbourne, E.D., Laver, W.G., Schulman, J.L. and Webster, R.G., 1968, J. Virol. 2, 281.

Köhler, G. and Milstein, C., 1975, Nature 256, 495.

Nakajima, S. and Kendal, A.P., 1981, Virology 113, 656.

Osterhaus, A.D.M.E., Van Wezel, A.L., Van Steenis, G., Drost, G.A. and Hazendonk, T.G., 1981, Intervirology 16, 218.

Osterhaus, A.D.M.E., Van Wezel, A.L., Hazendonk, T.G., UytdeHaag, F.G.C.M., Van Asten, J.A.A.M. and Van Steenis, G., 1983, Intervirology 20, 129.

Pereira, M.S. and Chakraverty, P., 1982, J. Hyg. (Camb.) 88, 501.

Skehel, J.J., Douglas, A.R., Wilson, I.A. and Wiley, D.C., 1982, in: Virus Persistence, eds. Mahy, B.W.J., Minson, A.C. and Darby, G.K. (Cambridge University Press, Cambridge) p. 215.

Smith, W., Andrewes, C.H. and Laidlaw, P.P., 1933, Lancet ii, 66.

Sokal, R.R. and Sneath, P.H.A., 1963, in: Principles of Numerical Taxonomy (W.H. Freeman, San Francisco) p. 305.

Takatsy, G. and Hamar, M., 1955, Acta Microbiol. Hung. 3, 203.

Webster, R.G., Kendal, A.P. and Gerhard, W., 1979, Virology 96, 258.

Wcbster, R.G., Laver, W.G., Air, G.M. and Schild, G.C., 1982, Nature 296, 115.

Yamazi, Y., Suzuki, H., Watari, E., Takeuchi, Y., Ohya, A., Satsuta, K., Yunoki, H., Supawadee, J., Chatiyanonda, K. and Thongcharoen, P., 1984, J. Jpn. Assoc. Infect. Dis. 58, 113. 